\title{
D-OPTIMALITY AND DL-OPTIMALITY CRITERIA FOR INCOMPLETE BLOCK DESIGNS
}

\author{
A. V. OLADUGBA AND M. S. MADUKAIFE \\ (Received 26, March 2009; Revision Accepted 3, June 2009)
}

\begin{abstract}
The adequacy of an experimental design can be determined from the information matrix. The D-optimality criterion is based on the determinant of the information matrix $\mathrm{M}(\xi)(\xi$ is any design measure) of a design, that is it maximizes the determinant of the information matrix $\mathrm{M}(\xi)$ or, equivalently, minimizes the determinant of inverse of the information matrix $M^{-1}(\xi)$. There are cases where the information matrix $\mathrm{M}(\xi)$ of a design degenerates (that is, the determinant is zero). In this situation, we introduce the use of the loss of information matrix designated as $L(\xi)$ matrix. The loss of information matrix $L(\xi)$ is a symmetric positive definite matrix that has exactly the same diagonal elements as those of the information matrix $M(\xi)$ and the off-diagonal elements lying between zero and one. $D_{\mathrm{L}}$-optimality criterion measures the determinant of the loss of information matrix.In this paper, we consider the correspondence between the $D$-and $D_{L}$-optimality criteria, that is whether a D-optimal design is also $D_{L}$-optimal, in a block or more than one block using a regular and irregular experimental region. An optimal design is selected with the aid of the combinatorial algorithm developed by Onukogu and Iwundu (2008). Breaking of ties existing in Doptimality criterion using the $D_{L}$-optimality criterion is also considered.
\end{abstract}

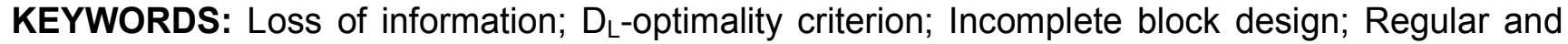
irregular experimental region.

\section{INTRODUCTION}

An optimality criterion is a single-valued measure that determines how good a design is, and it is maximized or minimized by an optimal design. The information-based criteria that are normally used are the D-, A-, E- and G-optimality, which are all related to the information matrix $\mathrm{M}(\xi)$ of the design. This $\mathrm{M}(\xi)$-matrix is important because it is proportional to the inverse of the variance-covariance matrix for the least-squares estimates of the parameters of the model. Roughly, a good design should "minimize" the variance of the inverse of the information matrix, which is the same as maximizing the information matrix. Indeed, the D-optimality criterion is based on the determinant of the information matrix of the design, which is the same as the reciprocal of the determinant of the variance-covariance matrix for the least-squares estimates of the parameters of the model. In order words, the D-optimality criterion maximizes the determinant of the information matrix $\mathrm{M}(\xi)$ or, equivalently, minimizes the determinant of the inverse of the information matrix $\mathrm{M}^{-1}(\xi)$.

Now in a situation where the information matrix is degenerate, that is, the determinant of the information matrix is zero; we introduce the use of the loss of information matrix designated as $L(\xi)$ - matrix. The loss of information matrix is a $(p \times p)$ non-singular, positive definite matrix, whose diagonal elements are exactly the same as the diagonal elements of the information matrix and the off-diagonal elements lie between zero and one. The $D_{L}$-optimality criterion maximizes the determinant of the loss of information matrix $L(\xi)$.

A. V. Oladugba, Department of Statistics, University of Nigeria, Nsukka

M. S. Madukaife, Department of Statistics, University of Nigeria, Nsukka 
Relative loss of information in a design is defined as a measure on relative efficiency of a design. Kshirsagar (1957), shows that if in any design where every treatment is replicated the same number of times, the total relative loss of information is one less than the average number of blocks per replication, that is, $\left(\frac{b}{r}-1\right)$, and the total relative information on any treatment estimate is $(v-b / r)$ where $v$ is the number of treatments, $b$ is the number of blocks and $r$ is the number of replicates. Damaraju (1971) gives the relative loss of information on the treatment estimate of a design as $\left(r-\frac{\theta_{i}}{r}\right)$ where $r$ is the number of replications in the design and $\theta_{i}$ is the trace of the information matrix, also called C-matrix. Onukogu (1997) defines loss of information on confounded effect as the number of degree-of-freedom of the interactions confounded divided by the number of degree-of-freedom of such interactions available; where the number of degree of freedom confounded is equal to the number of blocks degree-of-freedom.

\section{FUNDAMENTAL CONCEPTS}

\section{Information Matrix}

Given a regression function $Y(\underline{x})=f\left(\underline{x}_{,} \underline{x}_{2} \ldots, \underline{x}_{n}\right)+e$, to obtain an N-point design, the design matrix, $\mathrm{X}$, and the information matrix, $\mathrm{M}(\xi)$, are given, respectively as

$$
\underset{N \times p}{X}=\left(\underline{x}_{i j}\right)=\left[\begin{array}{c}
\underline{x}_{1}^{\prime} \\
\dot{x_{2}^{\prime}} \\
\mathrm{M} \\
\underline{x}_{N}^{\prime}
\end{array}\right]=\left[\begin{array}{rrrr}
x_{11} & x_{12} & \mathrm{~K} & x_{1 p} \\
x_{21} & x_{22} & \mathrm{~K} & x_{2 p} \\
\mathrm{M} & \mathrm{M} & \mathrm{O} & \mathrm{M} \\
x_{N 1} & x_{N 2} & \mathrm{~K} & x_{N p}
\end{array}\right] \text { and } M(\xi)=\left(m_{i j}\right)=\left[\begin{array}{rrrr}
m_{11} & m_{12} & \mathrm{~K} & m_{1 p} \\
m_{21} & m_{22} & \mathrm{~K} & m_{2 p} \\
\mathrm{M} & \mathrm{M} & \mathrm{O} & \mathrm{M} \\
m_{N 1} & m_{N 2} & \mathrm{~K} & m_{N p}
\end{array}\right]
$$

where

$$
m_{i i}=\sum_{k=1}^{N} x_{k i}^{2} \quad \forall i=i ; \quad m_{i j}=\sum_{k=1}^{N} x_{k i} x_{k j} \quad \forall \quad i \neq j
$$

and $p$ is the number of parameters in the function.

The normalized information matrix of a fixed-sized design, $\xi$, for $\underline{x}_{1}, \underline{x}_{2} \ldots \underline{x}_{n}$, is a (p×p) symmetric, positive-semi definite matrix, whose diagonal elements are the quotients of the sums of squares of the distances of the support points from the centre of the design with reference to a particular variate (parameter) and the number of support points $\mathrm{N}$ and the off-diagonal elements are the quotients of the sums of cross-products (i.e. distances of one support point from another with reference to any two variates (parameters)) and the number of support points $\mathrm{N}$.

$$
\text { i.e. } m_{i i}=\mathrm{N}^{-1} \sum_{k=1}^{N} x_{k i}^{2} \forall i=i ; m_{i j}=N^{-1} \sum_{k=1}^{N} x_{k i} x_{k j} \forall i \neq j
$$

If the information matrix, $\mathrm{M}(\xi)$, of an experiment is not degenerate, then the inverse of the determinant of the information matrix gives us the generalized variance of the design under consideration. For any design measure, $\xi$, the non-singular $(p \times p)$ information matrix, $M(\xi)$, is:

i) Symmetric, positive-semi definite; that is, for any vector (say) $\underline{u} \neq \underline{0}$ with real components, the quadratic form $\underline{u^{\prime}} \mathrm{M}(\xi) \underline{u} \geq 0$;

ii) Convex, that is, each element of the information matrix $\mathrm{M}(\xi)$ can be expressed as a convex combination, $\sum_{i=1}^{N} \lambda i \underline{x}_{i} \underline{x}_{i}^{\prime} \quad$ where $\underline{x}_{i} \in X, \lambda i>0, \Sigma \lambda i=1$;

iii) Continuous; that is, the entries that make up the information matrix, $M(\xi)$, are quadratic functions of quantitative variable which are continuous. 
iv) Differentiable; that is, $\frac{\partial M(\xi)}{\partial m_{i j}}=\frac{\partial M(\xi)}{\partial x_{k j}} \cdot \frac{\partial x_{k j}}{\partial m_{i j}} \neq 0$.

These are known as the analytical properties of the information matrix, $M(\xi)$, and the proof are shown in details in optimum experimental design literature such as Fedorov (1972), Silvey (1980) Pazman (1986), and Onukogu (1997) etc.

\subsection{Loss of information matrix}

The loss of information matrix, $L(\xi)$, of a fixed-size design, $\xi$, for $\underline{x}_{1}, \underline{x}_{2} \ldots, \underline{x}_{N}$ is a (pxp) symmetric, positive definite matrix whose diagonal elements are exactly the same as the diagonal elements of the information matrix and the off-diagonal elements lie between zero and one; that is; if the information matrix $\mathrm{M}(\xi)=\left(m_{i j}\right)$ for $\mathrm{i}, \mathrm{j}=1,2, \ldots, \mathrm{p}$ then the corresponding loss of information matrix $L(\xi)=\left(l_{i j}\right)$, where $l_{i j}=m_{i i}$ for all $i=j$ and $0 \leq l_{i j} \leq 1$ for all $i \neq j$.

$$
L(\xi)=\left(l_{i j}\right)=\left[\begin{array}{llll}
l_{11} & l_{12} & \Lambda & l_{1 p} \\
l_{21} & l_{22} & \mathrm{~K} & l_{2 p} \\
\mathrm{M} & \mathrm{M} & \mathrm{O} & \mathrm{M}^{3} \\
l_{p 1} & l_{p 2} & \Lambda & l_{p p}
\end{array}\right] \text { wherk } \xi_{i}=\sum_{x=1}^{N} x_{k i}^{2} \forall i=j ; l_{i j}=N^{-1}\left(\sum_{k=1}^{N} x_{k i} x_{k j}\right)^{2}\left(\sum_{k=1}^{N} x_{k i}^{2} x_{k j}^{2}\right)^{-1} \forall i \neq j \text { For any design }
$$

measure, $\xi$, the non-singular $(p \times p)$ loss of information matrix, $L(\xi)$, is:

i) Symmetric, positive definite; that is; for any vector (say) $\underline{u} \neq \underline{0}$ with real components, the quadratic form $\underline{u^{\prime}} \mathrm{L}(\xi) \underline{u}>0$;

ii) Convex; that is, each element of $L(\xi)$ can be expressed as a convex combination $\sum_{i=1}^{N} \lambda i \underline{x}_{i} \underline{x}_{i}^{\prime}$ where $\underline{x}_{i} \in X, \lambda_{i}>0, \sum \lambda i=1$

iii) Continuous; that is, the entries that make up $L(\xi)$ are quadratic functions of quantitative variable which are continuous;

iv) Differentiable; that is, $\frac{\partial L(\xi)}{\partial l_{i j}}=\frac{\partial L(\xi)}{\partial x_{k j}} \cdot \frac{\partial x_{k j}}{\partial l_{i j}} \neq 0$.

These are known as the analytical properties of the loss of information matrix, $L(\xi)$, and the proofs are shown in detail in Oladugba (2006).

\subsection{Regular and Irregular Experimental Region}

An experimental region is said to be regular if it takes the shape of any regular geometry like circle, rectangle, square, cube, cuboid, cone cylinder, etc; while any experimental region whose shape does not align with any regular geometry is known as an irregular experimental region. An example is a square with one of the quadrants cut-off.

\section{Derivation of the loss of information of a design}

There are many ways of deriving the loss of information of a design; but in this work, we used the means of the design matrix, $X$, formed with regard to the response function, $f(x)$, given, the entries of each treatment contrast will be added up and squared multiply by the inverse of sum of each of the treatment contrast squared divided by the size of the block or the size of the support point in the design $(\mathrm{N})$ considered, that is,

$$
L I\left(x_{i}\right)=\left(\underline{x}_{i}^{\prime} \underline{x}_{i}\right)^{-1} \sum_{j=1}^{b} x_{i}^{\prime} \underline{x}_{B j}\left(\underline{x}_{B j}^{\prime} \underline{x}_{B j}\right)^{-1} \underline{x}_{B j}^{\prime} \underline{x}_{i} ; i=1,2, . . p \text {. }
$$


Thus, considering the general block design, the derivation of the loss of information is obtained as follows: Given the general block model:

$$
f(x)=X_{t \underline{\alpha}}+X_{B \underline{\beta}}
$$

where $X_{t \underline{\alpha}}$ is an $(\mathrm{N} \times p)$ design matrix for the treatment parameters, $X_{B \underline{\beta}}=\left(\underline{x}_{i B}\right)$ is an $(\mathrm{N} \times \mathrm{B})$ block indicator matrix such that

$$
\underline{x}_{i B}=\left\{\begin{array}{l}
1, \text { if the }{ }^{\text {th }} \text { supporpointappearin the } \mathrm{d}^{\text {th }} \text { block } \\
0, \text { elsewhere }
\end{array}\right.
$$

$\underline{\alpha}$ and $\underline{\beta}$ are respectively $p$ and $b$ component vectors of the treatment and block effects, then; the loss of information (L.I) of the design when the block is greater than one is,

$$
L I .=\left(X_{t}^{t} X_{t}\right)^{-1} X_{t}^{t} X_{B}\left(X_{B}^{t} X_{B}\right)^{-1} X_{B}^{t} X_{t}=N^{1}\left(\sum_{i=1}^{N} x_{i k} x_{i B}\right)^{2}\left(\sum_{i=1}^{N} X_{i k}^{2} x_{i B}^{2}\right)^{-1}
$$

Assuming that there is no blocking or the block is one, then the loss of information (L.I) will be zero when the loss along the column of each factor is zero and if the loss is not equal to zero then the

loss of information, (L.I), is L.I. $=N^{-1}\left(\sum_{k=1}^{N} \underline{x}_{k i} \underline{x}_{k j}\right)^{2}\left(\sum_{k=1}^{N} \underline{x}_{k i}^{2} \underline{x}_{k j}^{2}\right)^{-1}$

\section{Numerical Illustration}

In this section, we consider whether a design that is D-optimal is also $\mathrm{D}_{\mathrm{L}}$-optimal: in a block or more than one block using a regular and irregular experimental region.

The optimal design here is selected with the aid of the combinatorial algorithm developed by Onukogu and Iwundu (2008). This is an iterative search technique which requires the identification of the best D-optimal design within a class and moves progressively from one class in a stage to another with a higher determinant value. In this technique, all the distinct support points that make up the experimental region are grouped into $\mathrm{H}$ concentric balls according to their distances from the centre of the region. The movement continues in the direction of higher determinant value until the D-optimal design is obtained.

4.1. Case One: (Regular Geometric Experimental Region)

Here, we consider a four-, five- and six-point designs for a bivariate linear response surface:

$f\left(x_{1}, x_{2}\right)=a_{00}+a_{10} x_{1}+a_{20} x_{2}+a_{12} x_{1} x_{2}+e$

defined in a regular experimental area shown below

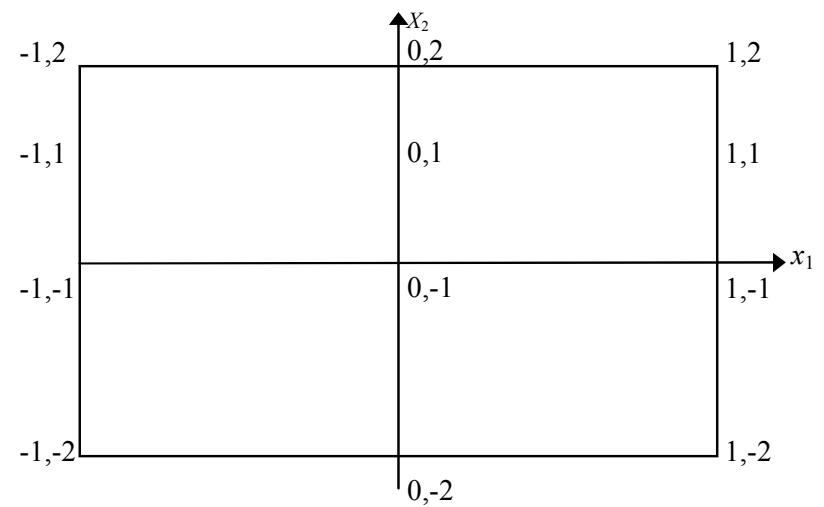

i.e. $\widetilde{X}=\left\{\underline{x}_{1}, \underline{x}_{2} ; \underline{x}_{1}=-1,0,1\right.$, and $\left.\underline{x}_{2}=-2,-1,1,2\right\}$.

Grouping the support points according to their distances from the centre, we have 


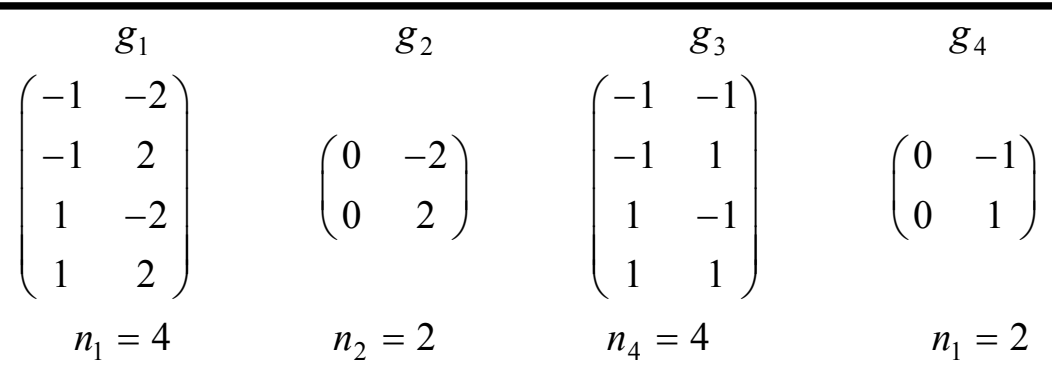

Without Blocking

\begin{tabular}{|c|c|c|c|c|c|c|c|c|c|}
\hline $\begin{array}{l}\text { Design } \\
\text { point }(\mathrm{N})\end{array}$ & $\begin{array}{l}\text { Step } \\
i\end{array}$ & j & $\begin{array}{l}\text { Class } \\
\text { size } \\
\text { (k) }\end{array}$ & $\begin{array}{l}g_{1} \\
4\end{array}$ & $\begin{array}{l}g_{2} \\
2\end{array}$ & $\begin{array}{l}g_{3} \\
4\end{array}$ & $\begin{array}{l}g_{4} \\
2\end{array}$ & $\begin{array}{l}\operatorname{det} \mathrm{M}\left(\xi_{\mathrm{N}}\right) \\
\text { Maximum } \\
\text { determinant in the jth } \\
\text { class } \mathrm{d}_{\mathrm{ij}}\end{array}$ & $\begin{array}{l}\text { det } L\left(\xi_{N}\right) \\
\text { Maximum determinant } \\
\text { in the jth class } c_{i j}\end{array}$ \\
\hline \multirow{2}{*}{4} & 1 & $\begin{array}{l}1 \\
2 \\
3 \\
4 \\
\end{array}$ & $\begin{array}{l}1 \\
1 \\
1 \\
1 \\
\end{array}$ & $\begin{array}{l}4 \\
0 \\
0 \\
0\end{array}$ & $\begin{array}{l}0 \\
4 \\
0 \\
0\end{array}$ & $\begin{array}{l}0 \\
0 \\
4 \\
0 \\
\end{array}$ & $\begin{array}{l}0 \\
0 \\
0 \\
4 \\
\end{array}$ & $\begin{array}{l}16.0^{* *}+11 \\
0.0 \\
1.0 \\
0.0 \\
\end{array}$ & $\begin{array}{l}16.0^{* *} \\
0.0 \\
1.0 \\
0.0\end{array}$ \\
\hline & 2 & $\begin{array}{l}1 \\
2 \\
3\end{array}$ & $\begin{array}{l}8 \\
16 \\
8 \\
\end{array}$ & $\begin{array}{l}3 \\
3 \\
3\end{array}$ & $\begin{array}{l}1 \\
0 \\
0\end{array}$ & $\begin{array}{l}0 \\
1 \\
0\end{array}$ & $\begin{array}{l}0 \\
0 \\
1\end{array}$ & $\begin{array}{l}4.0 \\
9.0^{*} \\
2.25 \\
\end{array}$ & $\begin{array}{l}8.9902 \\
10.5618^{*} \\
7.3042 \\
\end{array}$ \\
\hline \multirow{2}{*}{5} & 1 & $\begin{array}{l}1 \\
2 \\
3 \\
4\end{array}$ & $\begin{array}{l}4 \\
2 \\
4 \\
2\end{array}$ & $\begin{array}{l}5 \\
0 \\
0 \\
0\end{array}$ & $\begin{array}{l}0 \\
5 \\
0 \\
0\end{array}$ & $\begin{array}{l}0 \\
0 \\
5 \\
0\end{array}$ & $\begin{array}{l}0 \\
0 \\
0 \\
5\end{array}$ & $\begin{array}{l}13.1072 \\
0.0 \\
0.1892 \\
0.0\end{array}$ & $\begin{array}{l}15.9979^{\pi *} \\
0.0 \\
0.9996 \\
0.0\end{array}$ \\
\hline & 2 & $\begin{array}{l}1 \\
2 \\
3\end{array}$ & $\begin{array}{l}2 \\
4 \\
2\end{array}$ & $\begin{array}{l}4 \\
4 \\
4\end{array}$ & $\begin{array}{l}1 \\
0 \\
0\end{array}$ & $\begin{array}{l}0 \\
1 \\
0\end{array}$ & $\begin{array}{l}0 \\
0 \\
1\end{array}$ & $\begin{array}{l}9.8304 \\
10.6494^{*} \\
8.6016\end{array}$ & $\begin{array}{l}10.2398 \\
11.5592^{*} \\
8.7040\end{array}$ \\
\hline
\end{tabular}

\begin{tabular}{|c|c|c|c|c|c|c|c|c|c|}
\hline \multirow{2}{*}{6} & 1 & $\begin{array}{l}1 \\
2 \\
3 \\
4\end{array}$ & $\begin{array}{l}6 \\
1 \\
6 \\
1 \\
\end{array}$ & $\begin{array}{l}6 \\
0 \\
0 \\
0\end{array}$ & $\begin{array}{l}0 \\
6 \\
0 \\
0\end{array}$ & $\begin{array}{l}0 \\
0 \\
6 \\
0\end{array}$ & $\begin{array}{l}0 \\
0 \\
0 \\
6\end{array}$ & $\begin{array}{l}12.6420^{* *} \\
0.0 \\
0.7901 \\
0.0\end{array}$ & \begin{tabular}{|l}
15.9973 \\
0.0 \\
0.9973 \\
0.0
\end{tabular} \\
\hline & 2 & $\begin{array}{l}1 \\
2 \\
3\end{array}$ & $\begin{array}{l}8 \\
12 \\
8\end{array}$ & $\begin{array}{l}5 \\
5 \\
5\end{array}$ & $\begin{array}{l}1 \\
0 \\
0\end{array}$ & $\begin{array}{l}0 \\
1 \\
0\end{array}$ & $\begin{array}{l}0 \\
0 \\
1\end{array}$ & $\begin{array}{l}9.4815 \\
10.2716^{*} \\
8.2469\end{array}$ & $\begin{array}{l}11.1103 \\
12.2498^{*} \\
9.7216\end{array}$ \\
\hline
\end{tabular}

Notice: (") indicates an optimal design in a step and also (") indicates the local D-optimum for the $\mathrm{N}$-point design under consideration

\section{With Blocking}

Given the response function: $f\left(x_{1}, x_{2}\right)=a_{00}+a_{10} x_{1}+a_{20} x_{2}+a_{12} x_{2}+\sum_{j=1}^{b} b_{j} ;$ such that 
$\sum_{j=1}^{b} \beta_{j}=0$, where the block sizes are chosen arbitrarily.

\begin{tabular}{|c|c|c|c|c|c|c|c|c|c|c|}
\hline Design point $(\mathrm{N})$ & Step i & Class $\mathrm{j}$ & $\begin{array}{l}\text { Block } \\
\beta_{1}\end{array}$ & $\begin{array}{l}\text { Size } \\
\beta_{2}\end{array}$ & $\begin{array}{l}g_{1} \\
4\end{array}$ & $\begin{array}{l}g_{2} \\
2\end{array}$ & $\begin{array}{l}g_{3} \\
4\end{array}$ & $\begin{array}{l}g_{4} \\
2\end{array}$ & $\operatorname{det} \mathrm{M}\left(\xi_{5}\right)$ & det $L\left(\xi_{5}\right)$ \\
\hline \multirow{2}{*}{5} & 1 & $\begin{array}{l}1 \\
2 \\
3 \\
4\end{array}$ & $\begin{array}{l}3 \\
3 \\
3 \\
3 \\
3\end{array}$ & $\begin{array}{l}2 \\
2 \\
2 \\
2 \\
\end{array}$ & $\begin{array}{l}5 \\
0 \\
0 \\
0\end{array}$ & $\begin{array}{l}0 \\
5 \\
0 \\
0\end{array}$ & $\begin{array}{l}0 \\
0 \\
5 \\
0\end{array}$ & $\begin{array}{l}- \\
0 \\
0 \\
0 \\
5\end{array}$ & $\begin{array}{l}5.2429^{\pi *} \\
0.0 \\
0.3277 \\
0.0 \\
\end{array}$ & $\begin{array}{l}15.9750 \\
0.0 \\
0.9943 \\
0.0\end{array}$ \\
\hline & 2 & $\begin{array}{l}1 \\
2 \\
3\end{array}$ & $\begin{array}{l}3 \\
3 \\
3\end{array}$ & $\begin{array}{l}2 \\
2 \\
2\end{array}$ & $\begin{array}{l}4 \\
4 \\
4\end{array}$ & $\begin{array}{l}1 \\
0 \\
0\end{array}$ & $\begin{array}{l}0 \\
1 \\
0\end{array}$ & $\begin{array}{l}0 \\
0 \\
1\end{array}$ & $\begin{array}{l}1.3107 \\
2.9491^{*} \\
1.3107\end{array}$ & \begin{tabular}{|l}
10.1571 \\
$11.5125^{*}$ \\
8.6335
\end{tabular} \\
\hline
\end{tabular}

Case Two (Irregular Geometric Experimental Region)

We consider a five-point design for a bivariate linear response function defined in an irregular area shown below.

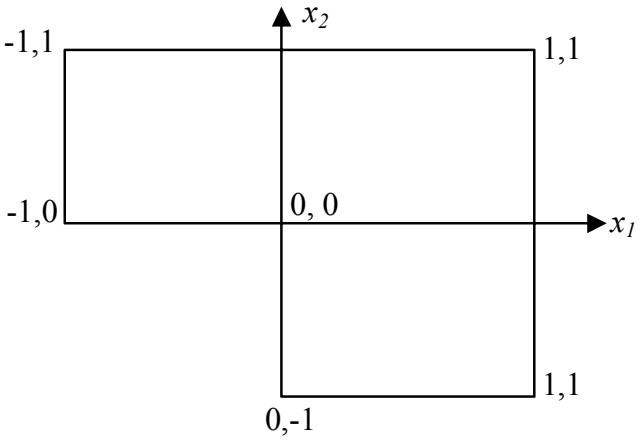

using a grid of 21 points, we form the following groups:

$$
\begin{aligned}
& \begin{array}{lllllll}
g_{1} & g_{2} & g_{3} & g_{4} & g_{5} & g_{6}
\end{array} \\
& \left(\begin{array}{cc}
1 & 1 \\
-1 & 1 \\
1 & -1
\end{array}\right)\left(\begin{array}{cc}
1 / 2 & 1 \\
-1 / 2 & 1 \\
1 & 1 / 2 \\
1 & -1 / 2 \\
1 / 2 & -1 \\
-1 & 1 / 2
\end{array}\right)\left(\begin{array}{cc}
-1 & 0 \\
1 & 0 \\
0 & -1 \\
0 & 1
\end{array}\right)\left(\begin{array}{cc}
1 / 2 & -1 / 2 \\
1 / 2 & 1 / 2 \\
-1 / 2 & 1 / 2
\end{array}\right)\left(\begin{array}{cc}
0 & -1 / 2 \\
0 & 1 / 2 \\
1 / 2 & 0 \\
-1 / 2 & 0
\end{array}\right)\left(\begin{array}{ll}
0 & 0
\end{array}\right) \\
& n_{1}=3 \quad n_{2}=6 \quad n_{3}=4 \quad n_{4}=3 \quad n_{5}=4 \quad n_{6}=1
\end{aligned}
$$


Without Blocking

\begin{tabular}{|c|c|c|c|c|c|c|c|c|c|c|c|}
\hline $\begin{array}{l}\text { Design } \\
\text { point (N) }\end{array}$ & $\begin{array}{l}\text { Step } \\
\text { I }\end{array}$ & $\begin{array}{l}\text { Class } \\
\mathrm{j}\end{array}$ & $\begin{array}{l}\text { Class } \\
\text { size }_{(\mathrm{k})}\end{array}$ & $\begin{array}{l}g_{1} \\
3\end{array}$ & $\begin{array}{l}g_{2} \\
6\end{array}$ & $\begin{array}{l}g_{3} \\
4\end{array}$ & $\begin{array}{l}g_{4} \\
3\end{array}$ & $\begin{array}{l}g_{5} \\
4\end{array}$ & $\begin{array}{l}g_{6} \\
1\end{array}$ & $\operatorname{det} M\left(\xi_{N}\right)$ & $\operatorname{det} L\left(\xi_{N}\right)$ \\
\hline \multirow{3}{*}{4} & 1 & $\begin{array}{l}1 \\
2 \\
3 \\
4 \\
5 \\
6\end{array}$ & $\begin{array}{l}3 \\
15 \\
1 \\
3 \\
1 \\
1\end{array}$ & $\begin{array}{l}4 \\
0 \\
0 \\
0 \\
0 \\
0\end{array}$ & $\begin{array}{l}0 \\
4 \\
0 \\
0 \\
0 \\
0\end{array}$ & $\begin{array}{l}0 \\
0 \\
4 \\
0 \\
0 \\
0\end{array}$ & $\begin{array}{l}0 \\
0 \\
0 \\
4 \\
0 \\
0\end{array}$ & $\begin{array}{l}0 \\
0 \\
0 \\
0 \\
4 \\
0\end{array}$ & $\begin{array}{l}0 \\
0 \\
0 \\
0 \\
0 \\
4\end{array}$ & $\begin{array}{l}7.4024 \times 10^{-17} \\
8.8 \times 10^{-3^{*}} \\
\text { Singular }(0.0) \\
2.8916 \times 10^{-19} \\
\text { Singular }(0.0) \\
\text { Singular }(0.0)\end{array}$ & $\begin{array}{l}0.9844^{\text {** }} \\
0.0888 \\
\text { Singular }(0.0) \\
0.0024 \\
\text { Singular }(0.0) \\
\text { Singular }(0.0)\end{array}$ \\
\hline & 2 & $\begin{array}{l}1 \\
2 \\
3 \\
4 \\
5\end{array}$ & $\begin{array}{l}6 \\
4 \\
3 \\
4 \\
1\end{array}$ & $\begin{array}{l}3 \\
3 \\
3 \\
3 \\
3\end{array}$ & $\begin{array}{l}1 \\
0 \\
0 \\
0 \\
0\end{array}$ & $\begin{array}{l}0 \\
1 \\
0 \\
0 \\
0\end{array}$ & $\begin{array}{l}0 \\
0 \\
1 \\
0 \\
0\end{array}$ & $\begin{array}{l}0 \\
0 \\
0 \\
1 \\
0\end{array}$ & $\begin{array}{l}0 \\
0 \\
0 \\
0 \\
1\end{array}$ & $\begin{array}{l}6.25 \times 10^{-2} \\
2.5 \times 10^{-1^{\star \star}} \\
3.52 \times 10^{-2} \\
1.406 \times 10^{-1} \\
6.25 \times 10^{-2}\end{array}$ & $\begin{array}{l}0.6554 \\
0.5608 \\
0.5021 \\
0.4555 \\
0.4020\end{array}$ \\
\hline & 3 & $\begin{array}{l}1 \\
2 \\
3 \\
4 \\
5\end{array}$ & $\begin{array}{l}18 \\
22 \\
36 \\
48 \\
12\end{array}$ & $\begin{array}{l}2 \\
2 \\
2 \\
2 \\
2\end{array}$ & $\begin{array}{l}0 \\
1 \\
0 \\
0 \\
0\end{array}$ & $\begin{array}{l}2 \\
1 \\
1 \\
1 \\
1\end{array}$ & $\begin{array}{l}0 \\
0 \\
1 \\
0 \\
0\end{array}$ & $\begin{array}{l}0 \\
0 \\
0 \\
1 \\
0\end{array}$ & $\begin{array}{l}0 \\
0 \\
0 \\
0 \\
1\end{array}$ & $\begin{array}{l}6.25 \times 10^{-2^{*}} \\
6.25 \times 10^{-2^{*}} \\
8.8 \times 10^{-3} \\
3.52 \times 10^{-2} \\
1.56 \times 10^{-2}\end{array}$ & $\begin{array}{l}0.2346 \\
0.3077^{*} \\
0.2133 \\
0.1967 \\
0.1740\end{array}$ \\
\hline \multirow{2}{*}{5} & 1 & $\begin{array}{l}1 \\
2 \\
3 \\
4 \\
5\end{array}$ & $\begin{array}{r}24 \\
16 \\
12 \\
16 \\
4\end{array}$ & $\begin{array}{l}4 \\
4 \\
4 \\
4\end{array}$ & $\begin{array}{l}0 \\
0 \\
0 \\
0\end{array}$ & $\begin{array}{l}1 \\
0 \\
0 \\
0\end{array}$ & $\begin{array}{l}0 \\
1 \\
0 \\
0\end{array}$ & $\begin{array}{l}0 \\
0 \\
1 \\
0\end{array}$ & $\begin{array}{l}0 \\
0 \\
0 \\
1\end{array}$ & $\begin{array}{l}5.12 \times 10^{-2} \\
2.048 \times 10^{-11^{* \star}} \\
2.88 \times 10^{-2} \\
1.152 \times 10^{-1} \\
5.12 \times 10^{-2}\end{array}$ & $\begin{array}{l}0.7157^{\pi} \\
0.6358 \\
0.5819 \\
0.5400 \\
0.5074\end{array}$ \\
\hline & 2 & $\begin{array}{l}1 \\
2 \\
3 \\
4 \\
5\end{array}$ & $\begin{array}{l}24 \\
96 \\
48 \\
64 \\
16\end{array}$ & $\begin{array}{l}3 \\
3 \\
3 \\
3 \\
3\end{array}$ & $\begin{array}{l}0 \\
1 \\
0 \\
0 \\
0\end{array}$ & $\begin{array}{l}2 \\
1 \\
1 \\
1 \\
1\end{array}$ & $\begin{array}{l}0 \\
0 \\
1 \\
0 \\
0\end{array}$ & $\begin{array}{l}0 \\
0 \\
0 \\
1 \\
0\end{array}$ & $\begin{array}{l}0 \\
0 \\
0 \\
0 \\
1\end{array}$ & $\begin{array}{l}1.536 \times 10^{-1} \\
1.920 \times 10^{-1} \\
1.352 \times 10^{-1} \\
1.632 \times 10^{-1} \\
1.408 \times 10^{-1}\end{array}$ & $\begin{array}{l}0.3594 \\
0.4403^{*} \\
0.1352 \\
0.3055 \\
0.2875 \\
\end{array}$ \\
\hline
\end{tabular}

With Blocking

\begin{tabular}{|c|c|c|c|c|c|c|c|c|c|c|c|c|}
\hline $\begin{array}{l}\text { Design point } \\
\text { (N) }\end{array}$ & $\begin{array}{l}\text { Step } \\
\text { i }\end{array}$ & $\begin{array}{l}\text { Class } \\
\mathrm{j}\end{array}$ & $\begin{array}{l}\text { Block } \\
B_{1}\end{array}$ & $\begin{array}{l}\text { Size } \\
B_{2}\end{array}$ & $\begin{array}{l}g_{1} \\
3\end{array}$ & $\begin{array}{l}g_{2} \\
6\end{array}$ & $\begin{array}{l}9_{3} \\
4\end{array}$ & $\begin{array}{l}g_{4} \\
3\end{array}$ & $\begin{array}{l}g_{5} \\
4\end{array}$ & $\begin{array}{l}g_{6} \\
1\end{array}$ & $\operatorname{det} M\left(\xi_{5}\right)$ & $\operatorname{det} L\left(\xi_{5}\right)$ \\
\hline \multirow[t]{15}{*}{ r } & \multirow{5}{*}{1} & 1 & 3 & 2 & 4 & 1 & 0 & 0 & 0 & 0 & $2.05 \times 10^{-2}$ & $0.7152^{* *}$ \\
\hline & & 2 & 3 & 2 & 4 & 0 & 1 & 0 & 0 & 0 & $8.19 \times 10^{-2^{\star *}}$ & 0.6345 \\
\hline & & 3 & 3 & 2 & 4 & 0 & 0 & 1 & 0 & 0 & $1.15 \times 10^{-2}$ & 0.2978 \\
\hline & & 4 & 3 & 2 & 4 & 0 & 0 & 0 & 1 & 0 & $5.1 \times 10^{-3}$ & 0.5390 \\
\hline & & 5 & 3 & 2 & 4 & 0 & 0 & 0 & 0 & 1 & $2.05 \times 10^{-2}$ & 0.5063 \\
\hline & \multirow{5}{*}{2} & 1 & 3 & 2 & 3 & 0 & 2 & 0 & 0 & 0 & $819 \times 10^{-2 *}$ & 03591 \\
\hline & & 2 & 3 & 2 & 3 & 1 & 1 & 0 & 0 & 0 & $5.1 \times 10^{-3}$ & $04120^{*}$ \\
\hline & & 3 & 3 & 2 & 3 & 0 & 1 & 1 & 0 & 0 & $5.14 \times 10^{-2}$ & 0.3314 \\
\hline & & 4 & 3 & 2 & 3 & 0 & 1 & 0 & 1 & 0 & $1.15 \times 10^{-2}$ & 0.2952 \\
\hline & & 5 & 3 & 2 & 3 & 0 & 1 & 0 & 0 & 1 & $5.1 \times 10^{-3}$ & 0.2717 \\
\hline & \multirow[t]{5}{*}{3} & 1 & 3 & 2 & 2 & 0 & 3 & 0 & 0 & 0 & $2.05 \times 10^{-2^{x}}$ & 0.1215 \\
\hline & & 2 & 3 & 2 & 2 & 1 & 2 & 0 & 0 & 0 & $5.1 \times 10^{-3}$ & $0.2116^{*}$ \\
\hline & & 3 & 3 & 2 & 2 & 0 & 2 & 1 & 0 & 0 & $8.0 \times 10^{-3}$ & 0.1488 \\
\hline & & 4 & 3 & 2 & 2 & 0 & 2 & 0 & 1 & 0 & $2.05 \times 10^{-2^{*}}$ & 0.1292 \\
\hline & & 5 & 3 & 2 & 2 & 0 & 2 & 0 & 0 & 1 & $2.05 \times 10^{-2^{*}}$ & 0.1215 \\
\hline
\end{tabular}




\subsection{Numerical Illustration on the Breaking of Ties Existing in D-optimal Design}

A tie is said to exist in D-optimal design when two or more designs of with the same number of support points $\mathrm{N}$ have equal maximum determinant of the information matrices resulting in more than one D-optimal designs. In this section, we consider the case of using the $D_{L}$-optimality criterion to break the ties existing in D-optimal designs.

Given a bivariate quadratic surface

$$
f\left(x_{1}, x_{2}\right)=a_{00}+a_{10} x_{1}+a_{20} x_{2}+a_{12} x_{1} x_{2}+a_{11} x_{1}^{2}+a_{22} x_{2}^{2}+e
$$

defined in $\tilde{X}=\left\{x_{1}, x_{2} ; x_{1}=-1,0,1\right.$ and $\left.x_{2}=-2,-1,1,2\right\}$

to obtain a 7-point design, using the class 4120 and 4021 we have,

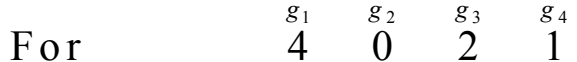

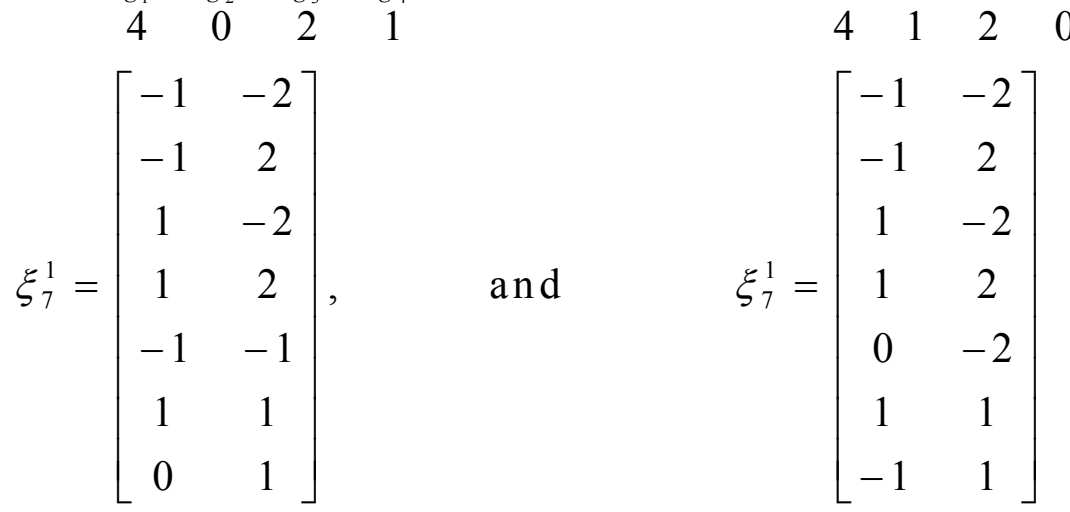

$\operatorname{Det} \mathrm{M}\left(\xi_{7}{ }^{1}\right)=\operatorname{Det} \mathrm{M}\left(\xi_{7}{ }^{2}\right)=1.0184$

Det $L\left(\xi_{7}{ }^{1}\right)=68.2001$ and Det $L\left(\xi_{7}{ }^{1}\right)=48.1160$ respectively. and also for

$$
f\left(x_{1}, x_{2}\right)=a_{00}+a_{10} x_{1}+a_{20} x_{2}+a_{12} x_{1} x_{2}+a_{11} x_{1}^{2}+a_{22} x_{2}^{2}+e
$$

defined in an irregular experimental area used section 4.2 to obtain a 6-point design; using the class 302010 and 302001 respectively.

We have

$$
\xi_{6}^{1}=\left[\begin{array}{cc}
1 & 1 \\
1 & -1 \\
-1 & 1 \\
-1 & 0 \\
1 & -1 \\
1 / 2 & 0
\end{array}\right], \quad \text { and } \quad \xi_{6}^{1}=\left[\begin{array}{cc}
1 & 1 \\
1 & -1 \\
-1 & 1 \\
-1 & 0 \\
0 & -1 \\
0 & 0
\end{array}\right]
$$

Det $\mathrm{M}\left(\xi_{6}{ }^{1}\right)=\operatorname{Det} \mathrm{M}\left(\xi_{6}{ }^{1}\right)=3.1 \times 10^{-3}$ while

Det $L\left(\xi_{6}{ }^{1}\right)=0.1009$ and Det $L\left(\xi_{6}{ }^{2}\right)=0.0939$ respectively.

\section{CONCLUSION}

In this work the D-optimality criterion (which has to do with the maximum determinant of the information matrix) was reviewed and a new optimality criterion known as the $D_{L}$-optimality criterion; which has to do with the maximum determinant of the loss of information matrix, was introduce.

The numerical demonstrations of this work are carried out in two experimental region that is the regular geometric experimental region $X^{0}=\left\{x_{1}, x_{2} ; x_{1}=-1,0,1\right.$ and $\left.x_{2}=-2,-1,1,2\right\}$ and the irregular geometric experimental region (using a grid of 21 points); for a bivariate linear response function; where the support point for any $\mathrm{N}$-point design is arranged in one homogeneous block or more than one homogeneous blocks. From the numerical illustration, it has been shown that there exists a correspondence between the D- and $D_{L}$ - optimality criteria for only a bivariate linear response function in a regular experimental region with or without blocking. For the bivariate linear response 
function in an irregular experimental region with or without blocking it is not always true. That is, a correspondence does not always exist between the $\mathrm{D}$ - and $D_{L}$ - optimality criteria. We note that the determinant of the loss of information matrix of a design is never zero even if the determinant of the information matrix of such design is equal to zero, unless for the case where all the support points of one of the factors under consideration is zero all through. break it.

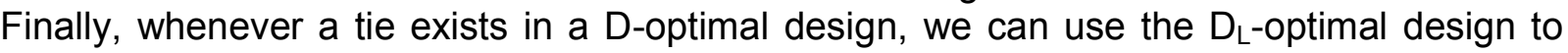

\section{REFERENCES}

Fedorov, V.V., 1972. Theory of Optimal Experiments. Academic Press Inc. New York.

Kshirsagar, A.M., 1952. A note on the total relative loss of information in any design. Cal, Stat. Assoc. Bill, 7.

Oladugba, A. V., 2006. On the correspondence between D-optimality and DL-optimality criteria. M.sc. Thesis, Department of Statistics, University of Nigeria, Nsukka.

Onukogu, I.B., 1997. Foundations of Optimal Exploration of Response Surface. Ephrata Press, Nsukka Nigeria.

Onukogu, I.B. and Iwundu, M.P., 2008. A Combinational Procedure for Constructing Optimal Experimental Designs. Statistica, to appear.

Pazman, A., 1986. Foundations of Optimum Experimental Design. D. Reidel Publishing Company.

Raghavarao, D., 1971. Constructions and Combinatorial Problems in Design of Experiments. John Wiley and Sons Inc. New York.

Silvey, S.D., 1980. Optimal design: An Introduction to the Theory for Parameter Estimation. Chapman and Hall Ltd, New York. 